\title{
AVALIAÇÃO DE CICLO DE VIDA E ECONÔMICA DE SISTEMA DE TRATAMENTO E REUSO DE EFLUENTES INTEGRANDO REATORES ANAERÓBIOS, WETLANDS CONSTRUÍDOS E OZONIZAÇÃO
}

\author{
CERENTINI P.1; PARANHOS, G.2; MACHADO³; Ê. L.; STOLZENBERG COLARES³; G. S
}

PALAVRAS-CHAVE: Saneamento Rural. Sustentabilidade. Energia renovável. Eutrofização. Fitorremediação.

\section{RESUMO}

A avaliação da sustentabilidade ambiental e dos custos econômicos de um sistema combinado de tratamento e reuso de efluentes composto por reatores anaeróbios (RAs), Wetlands construídos híbridos (WCs) e ozonização foram investigadas neste estudo. 0 software de Avaliação de Ciclo de Vida (ACV) SimaPro 8.04 foi aplicado nos dados de construção e monitoramento do sistema, que tratava águas residuais de um campus universitário. $A$ avaliação econômica foi realizada considerando os custos de construção, aquisição de equipamentos e operação. Em relação à construção do sistema, os RAs e WC2 apresentaram os maiores impactos potenciais devido ao polietileno de alta densidade e à fibra de vidro, respectivamente. Em relação à operação do sistema, a Ozonização foi responsável por 95\% dos impactos totais através da análise da rede, devido ao seu alto consumo de energia, sendo as categorias de impactos ambientais Aquecimento global, Energia não renovável, Efeitos respiratórios, Não cancerígeno e eutrofização foram as mais significativas. Quanto à avaliação econômica, o custo do tratamento considerando apenas os RAs foi estimado em $6,5 \mathrm{US} \$ / \mathrm{m}^{3}$, considerando que a integração com os WCs híbridos quase dobrou $\left(13,5\right.$ US $\left.\$ / \mathrm{m}^{3}\right)$, com o sistema combinado aumentou para $28,6 \mathrm{US} \$ / \mathrm{m}^{3}$. A maioria das despesas estava relacionada à demanda de energia e remoção e tratamento de lodo. Portanto, embora 0 sistema atinja os padrões locais para reutilização de águas residuais, estudos futuros devem investigar a geração local de energia renovável e materiais de construção alternativos para reduzir impactos e custos.

\section{LIFE CYCLE AND ECONOMIC EVALUATION OF EFFLUENT TREATMENT AND REUSE SYSTEM INTEGRATING ANAEROBIC REACTORS, CONSTRUCTED WETLANDS AND OZONIZATION}

KEYWORDS: Rural Sanitation. Sustainability. Bibliometric Analysis. Renewable Energy. Eutrophication. Phytoremediation.

\begin{abstract}
The evaluation of the environmental sustainability and economic costs of a combined system for wastewater treatment and reuse composed by anaerobic reactors (ARs), hybrid constructed wetlands (CWs) and ozonation were investigated in this study. The Life Cycle Assessment (LCA) software SimaPro 8.04 was applied on the data from the system construction and monitoring, which was treating wastewater from an university campus. Economic assessment was conducted considering construction, equipment acquisition and operation costs. In relation to system construction, ARs and CW2 presented most potential impacts due to high-density polyethylene and fiberglass, respectively. Regarding the system operation, Ozonation accounted for $95 \%$ of total impacts through network analysis, due to its high energy consumption, and the environmental impacts categories Global warming, Non-renewable energy, Respiratory effects, Non-carcinogenic and eutrophication were the most significant. Concerning the economic assessment, the treatment cost considering only the ARs was estimated in $6.5 \mathrm{US} \$ / \mathrm{m}^{3}$, considering the integration with the hybrid CWs it nearly doubled $\left(13.5 \mathrm{US} \$ / \mathrm{m}^{3}\right)$, with the combined system it increased to $28.6 \mathrm{US} \$ / \mathrm{m}^{3}$. Most expenses were related to energy demand and sludge removal and treatment. Therefore, although the system achieved local standards for wastewater reuse, future studies should investigate local generation of renewable energy and alternative construction materials to reduce impacts and costs.
\end{abstract}

\footnotetext{
1 Acadêmica do Curso de Engenharia Química - UNISC.

2 Acadêmica do Curso de Engenharia Química - UNISC

${ }^{3}$ Programa de Pós-graduação Programa de Pós-Graduação em Tecnologia Ambiental - Mestrado e Doutorado - PPGTA-UNISC
} 


\section{INTRODUÇÃO}

Em relação à atual crise de escassez de água, diversas tecnologias voltadas para o tratamento local e reaproveitamento de águas residuárias estão se tornando atrativas, principalmente quando o objetivo principal é o reaproveitamento não potável da água (MASMOUDI JABRI et al., 2019). Nesse contexto está o saneamento descentralizado, onde os estudos estão focados em minimizar o desperdício de recursos, consumo de energia e demanda de água potável, além de reduzir a geração de resíduos e, possivelmente, proporcionar recuperação de nutrientes e até geração de energia a partir de águas residuais (DELL'OSBEL et al., 2020).

E tendo como referência saneamento descentralizado sustentável, devem ser considerados os Wetlands Construídos (WCs), que provaram ser uma alternativa interessante para o tratamento descentralizado de águas residuais, devido à sua construção e manutenção simples (WU et al., 2015). Além disso, os sistemas WCs estão surgindo como uma alternativa interessante para a falta de saneamento básico nos países em desenvolvimento, principalmente quando aplicados após um tratamento primário como fossas sépticas, ao invés de coletar e transportar o esgoto para uma estação central de tratamento, o que é muito comum para residências rurais (DE SOUZA CELENTE et al., 2020). Além de tratar águas residuais, os WCs têm bom potencial para reutilizar água e nutrientes, podem tolerar alta variabilidade e promover o habitat de vida selvagem (WANG et al., 2017).

Assim sendo, a presente pesquisa considerou dois pontos importantes da sustentabilidade no saneamento, as questões ambientais e econômicas, considerando que a continuidade em futuros trabalhos também deverá incluir o aspecto social, especialmente através de ferramentas que incluíssem as Avaliações de Ciclo de Vida (ACV) e Econômica, que poderão embasar futuros estudos de ACV Social em futuras pesquisas.

\section{FUNDAMENTAÇÃO TEÓRICA}

No meio rural, integrar sistemas de WCs com reatores anaeróbios é uma tendência para o desenvolvimento sustentável, que permite a recuperação de materiais, energia e nutrientes, apresentando alta eficiência na remoção de nutrientes, menor pressão ambiental (LUTTERBECK et al., 2017), fácil manutenção e operação, boa integração com a paisagem e promoção da biodiversidade local (PELISSARI et al., 2017).

Apesar dos benefícios ambientais e econômicos obtidos com o uso dos WCs, altas cargas poluentes, principalmente de Nitrogênio Total Kjeldahl (NTK) e fósforo total (P), que apresentam grandes exigências de superfície, estão promovendo a necessidade de integração desses sistemas com outros processos de prétratamento e polimento final para atender aos requisitos legais e normativos. Ao integrar os WCs com processos oxidativos avançados (POAs), por exemplo, a detoxificação e desinfecção das águas residuais pode ser alcançada e, assim, permitir a reutilização das águas residuais tratadas (HORN et al., 2014).

Como exemplo de integração de WCs com POAs, pode ser citada a integração de uma unidade de ozonização (que em presença de coadjuvantes como a radiação UVC pode ser um POA), e que tem potencial para promover a remoção da cor da água, aspecto fundamental para o reúso da água (COLARES et al., 2019). Vários pesquisadores investigaram diferentes configurações de WCs, desde sistemas muito simples até a combinação de WCs com sistemas mais complexos e sofisticados, como WCs aerados artificialmente (WU et al., 2014), WCs e microalgas (DE SOUZA CELENTE et al., 2019). Trabalhos com estas combinações podem também ser 
exemplificados com WCs e ozonização (TRIPATHI e TRIPATHI 2011; HORN et al., 2014); e unidade de WC associada à desinfecção UV (ÁLVAREZ et al., 2017).

A análise dos potenciais impactos ambientais relativos às tecnologias de tratamento de efluentes deve auxiliar os tomadores de decisão na escolha da melhor alternativa de tratamento de efluentes ou na realização de modificações em sistemas já existentes que possam reduzir os impactos ambientais relacionados a estes (RESENDE et al., 2019). A Avaliação do Ciclo de Vida (ACV) é uma ferramenta concebida inicialmente para quantificar a demanda/consumo de recursos e também os impactos ambientais associados a um determinado produto. No entanto, também pode ser eficaz para avaliar os potenciais impactos ambientais das estruturas de saneamento (COROMINAS et al., 2013), e contribuir para um melhor desempenho global em cada fase do ciclo de vida, fornecendo, portanto, um indicativo para a sustentabilidade de um sistema estudado (LUTTERBECK et al., 2017).

Nesse contexto, a ACV é uma ferramenta essencial para tomadores de decisão e demais profissionais para a gestão integrada da água e o desenvolvimento sustentável, por permitir a comparação entre diferentes tecnologias de tratamento e melhorar a sustentabilidade das atuais unidades de saneamento. Masmoudi Jabri et al. (2019) investigaram um sistema de tratamento composto por dois reatores de biofilme de leito móvel (MBBR) integrados com lâmpadas UV para o tratamento de água cinza de uma residência e visando a reutilização local não potável. Os autores avaliaram os impactos ambientais de cada uma das unidades de tratamento por meio da ACV. No entanto, nenhuma análise sobre os custos de construção e operação do sistema de tratamento foi realizada. Diante disso, deve-se estar ciente de que a implantação de um sistema depende também dos custos associados ao tratamento de efluentes. Esse aspecto é especialmente importante em países em desenvolvimento que podem sofrer com a escassez de água e têm recursos limitados para investimentos em saneamento básico.

Portanto, a aplicação da ACV e da Análise de custeio são importantes para avaliar a integração inovadora de tecnologias, bem como, melhorar o tratamento de efluentes visando o reuso local da água. Assim sendo, este estudo investigou cada uma das etapas de tratamento do sistema integrado, que foi composta por reatores anaeróbios, WCs híbridos e unidade de ozonização, associando avaliações quanto aos impactos ambientais e custos relacionados à construção e operação do sistema.

\section{MATERIAIS E MÉTODOS}

\subsection{Monitoramento do sistema de tratamento}

O sistema combinado foi construído e operado na Estação de Tratamento de Efluentes (ETE) do Campus da Universidade de Santa Cruz do Sul (UNISC), por um período de 12 meses (2017). A ETAR tem capacidade para tratar $360 \mathrm{~m} 3 \mathrm{~d}-1$ de efluente urbano ou atender 18.000 pessoas. A maior parte do efluente gerado no campus universitário é proveniente de banheiros e mictórios, com vazão média de $8 \mathrm{~m}^{3} \mathrm{~h}-1$, variando de 70 a $120 \mathrm{~m}{ }^{3} \mathrm{~h}-1$ nos horários de pico. A água residual bruta (após passar gradeamento e remoção de areia) foi bombeada para os reatores anaeróbicos com uma bomba de 1 HP que foi ligada por 5 minutos por carregamento.

0 sistema de tratamento combinado foi composto por 4 reatores anaeróbios (1 tanque de sedimentação e 3 biodigestores), 3 WCs híbridos em estágios sequenciais e uma unidade de ozonização em escala de bancada. 
Os reatores anaeróbios foram compostos por 4 tanques de polietileno de alta densidade (PEAD) com volume de trabalho de 100 L cada, com 7 dias de tempo de retenção hidráulica (TRH) e peso de 10,8 $\mathrm{kg}$. Os reatores foram conectados através de mangueiras de cloreto de polivinila (PVC) com diâmetro de $20 \mathrm{~mm}$ e utilizando mangueiras de mais de 2 metros (1,4 kg). Os RAs foram alimentados semanalmente com água residuária do tanque de equalização, após passar apenas pelas unidades de peneiramento e remoção de areia. A Figura 1 apresenta um gráfico simplificado do sistema desenvolvido.

Figura 1 - Sistema de tratamento integrando RAs + WCs híbridos + $\mathrm{O}_{3}$ estudado para aplicação de ACV ferramenta SimaPro 8.04.

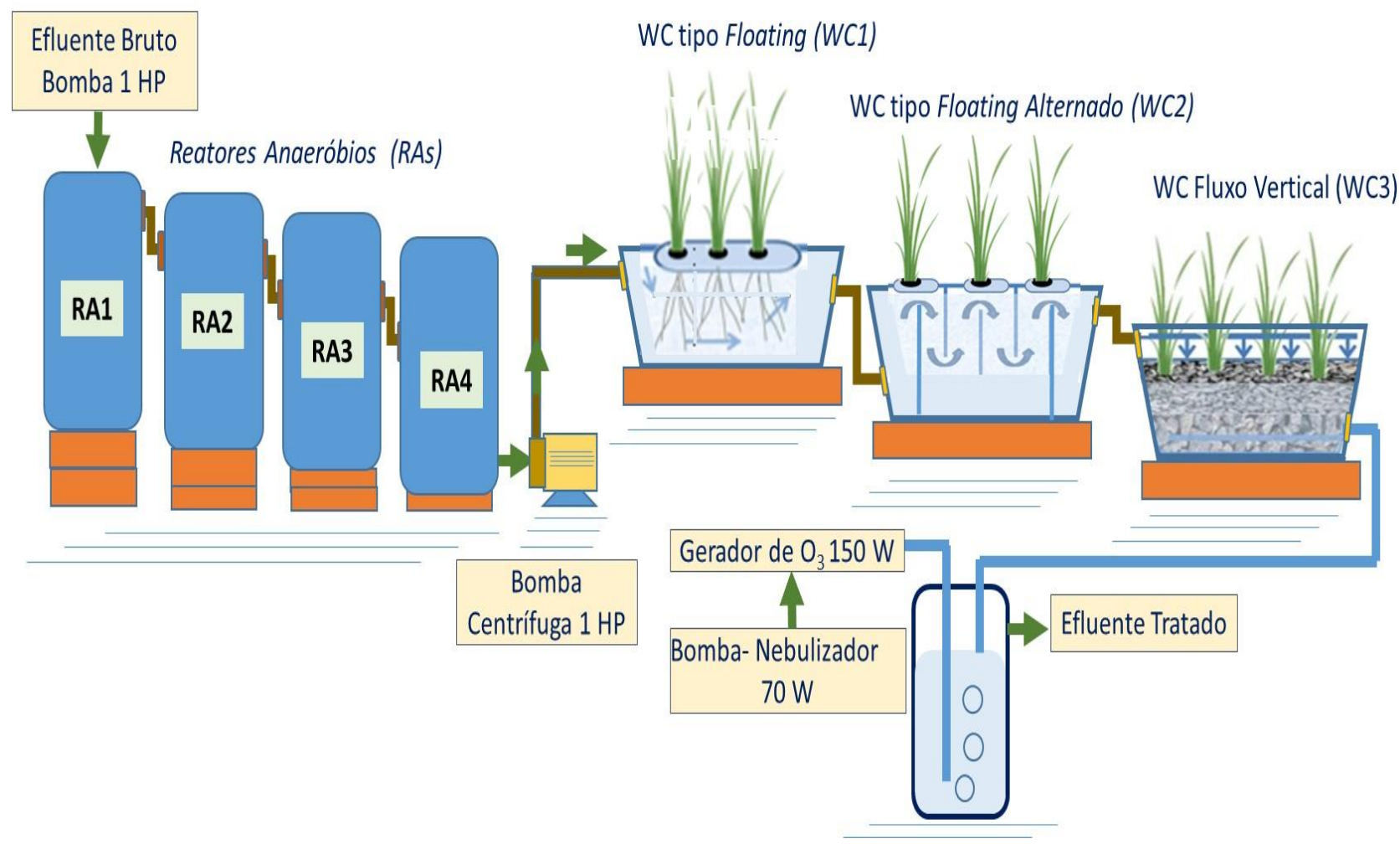

A segunda etapa do tratamento consistiu em um sistema WCs híbridos composto por duas caixas de PEAD (WC tipo Floating e WC Fluxo Vertical) e uma caixa de fibra de vidro (WC tipo Floating Alternado). 0 TRH de cada uma das caixas foi de 7 dias, totalizando 21 dias. Semelhante aos RAs, o sistema de alimentação, amostragem e determinações analíticas foram realizadas uma vez por semana durante 12 meses e as análises realizadas na coleta. Na primeira unidade (WC tipo Floating), plantas de Hymenachne grumosa (Nees) Zuloaga foram colocadas em tubos de PVC no sistema flutuante (9 plantas $\left./ \mathrm{m}^{2}\right)$, e os vazios entre as plantas foram preenchidos com alfaced'água (Pistia stratiotes L.) e lentilha-d'água (Spirodella sp.) a fim de cobrir a superfície da água e prevenir a proliferação de mosquitos. Tubos de PVC, totalizando cerca de $2 \mathrm{~m}$ (ou 0,10 kg), fizeram a drenagem.

A última unidade de tratamento foi um gerador de ozônio RADSAT com capacidade de geração de 2 g $\mathrm{O}_{3} \mathrm{~h}$ 1. A taxa de aplicação foi de $160 \mathrm{mg} \mathrm{O}_{3} \mathrm{~L}^{-1}$ ao longo de uma hora, visando principalmente a remoção da cor e garantia de desinfecção da água residuária para reuso doméstico não potável. 0 sistema foi monitorado por 12 
meses, com coleta e análise semanal de amostras. Mais informações sobre o projeto do sistema e as condições de operação podem ser encontradas em Colares et al. (2019).

\subsection{Avaliação do Ciclo de Vida para construção e operação de tratamento}

A aplicação de ACV foi realizada para um fluxo de referência de 20 anos, com base na vida útil do sistema de tratamento e nas pesquisas desenvolvidas por Garfí et al. (2017). A unidade funcional foi definida como 100 litros de água residual bruta tratada por semana, que era a quantidade de água tratada pelo sistema combinado durante seu monitoramento. Os procedimentos metodológicos seguiram a Associação Brasileira de Normas Técnicas ABNT NBR 14044 (2009) - Gestão Ambiental: Avaliação do Ciclo de Vida - Princípios e Estrutura e ABNT NBR ISO 14040 (2009) - Gestão Ambiental: Avaliação do Ciclo de Vida - Requisitos e Orientações.

O software utilizado para o ACV foi o SimaPro versão 8.04 e o banco de dados utilizado no ACV foi o Ecoinvent 3 com método de impacto IMPACT 2002+ do Swiss Federal Institute of Technology. Foram considerados os dados de Caracterização, Normalização e Pontuação Única.

A abordagem metodológica da avaliação "Impact 2002+" permite a implementação viável de um ponto médio em uma abordagem combinada de danos (LUTTERBECK et al., 2020). Conecta todos os tipos de resultados de inventário do ciclo de vida (fluxos elementares e outras intervenções), por vários níveis médios para diferentes categorias de danos (VARANDA et al., 2011). Os impactos ambientais foram avaliados de acordo com 15 indicadores diferentes: carcinogênico (CA), não carcinogênico (NCA), inorgânico respiratório (RI), radiação ionizante (IR), destruição da camada de ozônio (OD), orgânicos respiratórios (RO), ecotoxicidade aquática ( $A E$ ), ecotoxicidade terrestre (TE), acidez terrestre (TA-N), ocupação do solo (LO), acidificação aquática (AA), eutrofização aquática (AE), aquecimento global (GW), energia não renovável (NRE) e extração mineral (ME) (Muñoz et al., 2006).

Neste estudo, a avaliação da operação de cada etapa de tratamento (RAs + híbridos CWs e 03) e o descarte sem tratamento da água residual bruta foram consideradas em diferentes cenários. Além disso, a ACV do sistema de tratamento foi segregada em construção e operação, a fim de identificar as unidades mais impactantes em cada uma delas.

\section{RESULTADOS E DISCUSSÃO}

\subsection{Caracterização de dados para inventário de ciclo de vida}

Para a ACV da construção e operação do sistema combinado, um resumo dos materiais e insumos usados são apresentados na Tabela 1. Devido à ausência de geradores de ozônio na base de dados Ecoinvent, foi considerado que o gerador de ozônio utilizado no presente estudo (RADAST 2C) foi composto por poliestireno (caixa de plástico), aço inoxidável-cromo (18/8), alumínio e cobre. 
Tabela 1. Inventário de insumos de operação e materiais de construção e equipamentos de cada uma das etapas de tratamento do sistema combinado.

\begin{tabular}{|c|c|c|c|c|c|c|}
\hline “ & Unidade & RAs & CW1 & WC2 & WC3 & $\mathbf{O}_{3}$ \\
\hline \multicolumn{7}{|l|}{ Construção } \\
\hline Suporte de escada de metal & kg & - & 20 & 10 & 5 & - \\
\hline Polietileno de Alta Densidade & $\mathrm{kg}$ & 120 & 40 & - & 40 & - \\
\hline Fibra de Vidro & kg & - & - & 40 & - & - \\
\hline Tubos de PVC & kg & 1,8 & 1.5 & 0.5 & 1 & - \\
\hline Espuma de Poliuretano & kg & - & 0,17 & 0,17 & - & - \\
\hline Seixos & kg & - & - & - & 50 & - \\
\hline Brita n.2 & kg & - & - & - & 100 & - \\
\hline Vidro Borossilicato (Béquer) & kg & - & - & - & - & 0,06 \\
\hline Poliestireno & $\mathrm{kg}$ & - & - & - & - & 3.5 \\
\hline Aço Inoxidável & kg & - & - & - & - & 0,1 \\
\hline Cobre & kg & - & - & - & - & 0,03 \\
\hline Alumínio & kg & - & & - & - & 0,5 \\
\hline \multicolumn{7}{|l|}{ Operação } \\
\hline Consumo de Energia & $\begin{array}{c}\text { kWh } \\
\text { /semana }\end{array}$ & 0,124 & 0,062 & - & - & 0.67 \\
\hline Poda - Biomassa & $\mathrm{hr} /$ ano & - & 1 & - & 2 & - \\
\hline Água (resfriamento) & $\begin{array}{c}\mathrm{m}^{3} / \text { sema } \\
\text { na }\end{array}$ & - & - & - & - & 0.35 \\
\hline Remoção de Lodo & $\begin{array}{c}\mathrm{m}^{3} / 5 \\
\text { anos }\end{array}$ & 0,16 & - & - & - & - \\
\hline
\end{tabular}

Em relação às remoções estimadas de poluentes, cada etapa do sistema de tratamento integrado é apresentada considerando como unidade funcional 100 litros de água residual bruta (tratada por semana) em uma vazão de referência de 20 anos, tendo a massa $(\mathrm{kg})$ das taxas de carregamento de poluentes determinada no período de operação do sistema (Tabela 2). Todos os parâmetros apresentados na Tabela 2 foram considerados na ACV de operação do sistema. A metodologia aplicada (vida útil de 20 anos para o sistema) foi semelhante às conduzidas por Garfí et al. (2017) e Flores et al. (2019).

Tabela 2. Caracterização das diferentes etapas da unidade de tratamento de efluentes estudada.

\begin{tabular}{|c|c|c|c|c|c|c|}
\hline \multirow[t]{2}{*}{ Parameter } & $\begin{array}{c}\text { Concentração } \\
\text { de Entrada } \\
\left(\mathrm{mgL}^{-1}\right)\end{array}$ & $\begin{array}{c}\text { Concentração } \\
\text { de Saída } \\
\left(\mathrm{mgL}^{-1}\right)\end{array}$ & $\begin{array}{c}\text { Fluxo de } \\
\text { Referência } \\
\text { kg } \\
\left(20 \text { anos }^{-1}\right)\end{array}$ & $\begin{array}{c}\text { Concentração } \\
\text { de Entrada } \\
\left(\mathrm{mgL}^{-1}\right)\end{array}$ & $\begin{array}{c}\text { Concentração } \\
\text { de Saída } \\
\left(\mathrm{mgL}^{-1}\right)\end{array}$ & $\begin{array}{c}\text { Fluxo de } \\
\text { Referência } \\
\text { kg } \\
\left(20 \text { anos }^{-1}\right)\end{array}$ \\
\hline & \multicolumn{3}{|c|}{ EFLUENTE BRUTO } & \multicolumn{3}{|c|}{ RAs } \\
\hline $\mathrm{DBO}_{5}$ & - & $192.2 \pm 66.1$ & 20 & $192 \pm 66$ & $112 \pm 59$ & 11.66 \\
\hline DQO & - & $481 \pm 266$ & 50.1 & $481 \pm 266$ & $96.8 \pm 14.2$ & 10.07 \\
\hline сOT & - & $140 \pm 49.9$ & 14.6 & $140 \pm 49$ & $30 \pm 16$ & 3.12 \\
\hline
\end{tabular}




\begin{tabular}{|c|c|c|c|c|c|c|}
\hline CT & - & $224 \pm 23.7$ & 23.3 & $224 \pm 23$ & $173 \pm 21$ & 17.99 \\
\hline $\mathrm{N}-\mathrm{NH}_{3}$ & - & $81.6 \pm 9.8$ & 8.5 & $82 \pm 9$ & $94.4 \pm 12$ & 9.82 \\
\hline NT & - & $92.5 \pm 25.3$ & 9.6 & $92 \pm 25$ & $122 \pm 27$ & 12.69 \\
\hline \multirow[t]{3}{*}{ P Solúvel } & - & $3.9 \pm 0.6$ & 0.4 & $3.9 \pm 0.6$ & $4.96 \pm 1.1$ & 0.52 \\
\hline & \multicolumn{3}{|c|}{ WC1 } & \multicolumn{3}{|c|}{ WC2 } \\
\hline & $112.1 \pm 59$ & $64.9 \pm 36.1$ & 6.75 & $64.9 \pm 36.1$ & $140 \pm 19.9$ & 14.6 \\
\hline DQO & $96.8 \pm 14.2$ & $62.3 \pm 14.1$ & 6.48 & $62.3 \pm 10.1$ & $68.7 \pm 43.1$ & 7.15 \\
\hline COT & $30 \pm 16$ & $4.26 \pm 4.08$ & 0.44 & $4.3 \pm 4.1$ & $11.3 \pm 8.7$ & 1.18 \\
\hline CT & $173 \pm 21$ & $110.9 \pm 26.4$ & 11.53 & $111 \pm 26.4$ & $91.0 \pm 11.9$ & 9.464 \\
\hline $\mathrm{N}-\mathrm{NH}_{3}$ & $94.4 \pm 12$ & $65.2 \pm 22.2$ & 6.78 & $65.2 \pm 22.2$ & $37 \pm 16.3$ & 3.85 \\
\hline NT & $122 \pm 27$ & $83.9 \pm 37.1$ & 8.73 & $83.9 \pm 37.1$ & $51.1 \pm 24.2$ & 5.32 \\
\hline \multirow[t]{2}{*}{ P Solúvel } & $4.96 \pm 1.14$ & $4.5 \pm 4.1$ & 0.47 & $4.5 \pm 4.1$ & $4.1 \pm 0.81$ & 0.42 \\
\hline & \multicolumn{3}{|c|}{ CW3 } & \multicolumn{3}{|c|}{$\mathrm{CW} 3+\mathrm{O}_{3}$} \\
\hline $\mathrm{DBO}_{5}$ & $140.4 \pm 19.9$ & $77 \pm 62.8$ & 8.01 & $77 \pm 62.8$ & $30 \pm 24$ & 3.12 \\
\hline DQO & $68.7 \pm 43.1$ & $30.6 \pm 19.9$ & 3.18 & $30.6 \pm 19.9$ & $86 \pm 84$ & 8.94 \\
\hline СОт & $11.3 \pm 8.7$ & $17 \pm 17.6$ & 1.77 & $17 \pm 17.6$ & $29.1 \pm 3.2$ & 3.03 \\
\hline $\mathrm{CT}$ & $91.0 \pm 11.9$ & $119 \pm 62.9$ & 12.33 & $118 \pm 63$ & $93.9 \pm 2.57$ & 9.77 \\
\hline $\mathrm{N}-\mathrm{NH}_{3}$ & $37.02 \pm 16.3$ & $3.25 \pm 3.82$ & 0.34 & $3.2 \pm 3.8$ & $4.49 \pm 1.22$ & 0.47 \\
\hline NT & $51.1 \pm 24.2$ & $6.5 \pm 8.7$ & 0.68 & $6.5 \pm 8.7$ & $5.89 \pm 1.30$ & 0.61 \\
\hline P Solúvel & $4.1 \pm 0.8$ & $1.3 \pm 1.3$ & 0.14 & $1.3 \pm 1.3$ & $3.20 \pm 0.65$ & 0.33 \\
\hline
\end{tabular}

Fonte: Adaptado a partir de Colares et al. (2019).

A biomassa seca e úmida de macrófitas foi estimada em 57,26 kg e 272,68 kg para o primeiro e terceiro WCs, respectivamente. 0 sistema foi capaz de atingir a desintoxicação total do efluente, em ensaios agudos realizados com o microcrustáceo Daphnia magna (STRAUS, 1820), antes mesmo da unidade de ozonização. 0 efluente tratado poderia ser classificado como Classe 2 pelas normas brasileiras NBR 13969/97, o que permitiria sua aplicação na lavagem de pisos, irrigação de jardins, manutenção e canais para fins paisagísticos. Embora os principais objetivos da aplicação de ozônio sejam a redução da cor (420 nm) e a desinfecção, esses aspectos não são considerados no Inventário de Ciclo de Vida (ICV).

\subsection{ACV da construção do sistema}

A avaliação dos impactos das diferentes unidades que compõem o sistema integrado consistiu na classificação dos processos em diferentes categorias de impacto ambiental (método Impact 2002+) seguida da caracterização. Em seguida, com o objetivo de perceber a magnitude do impacto causado pelos problemas ambientais de um ponto de vista global (VARANDA et al., 2011), foi realizada a normalização dos resultados inventariados. 
Em relação à construção do sistema RAs + WCs + 03, a Figura 2 apresenta os materiais mais importantes de acordo com a análise ACV, quanto à caracterização dos impactos (Figura 2A) e normalização (2B). Pela Figura 2, é possível notar que a unidade de ozonização $\left(\mathrm{O}_{3}\right)$ apresentou os menores impactos em relação à outra unidade de tratamento. Por outro lado, os reatores anaeróbios (ARs) e a segunda unidade de tratamento de zonas úmidas (WC2) apresentaram os maiores impactos em relação à fase de construção.

Com base nos resultados de normalização apresentados na Figura 2, pode-se observar que, considerando a magnitude dos impactos, quatro categorias foram responsáveis pelos maiores encargos ambientais: $C A, N-C A$, RI, GW e NRE. A partir dos dados obtidos, verificou-se que as RAs foram as unidades mais impactantes nas categorias de $\mathrm{CA}\left(37,4 \mathrm{~kg}\right.$ dos $73,4 \mathrm{~kg}$ de $\mathrm{CH}_{2} \mathrm{H}_{3} \mathrm{Cl}$ eq do total do sistema) e energia não renovável $\left(9,42 \mathrm{E}^{3}\right.$ de 2,28E4 MJ primária). Por outro lado, a unidade WC2 foi responsável pelos maiores impactos ambientais nas categorias de RI (0,268 de 0,614 kg PM2,5 eq) e GW (356 de $803 \mathrm{~kg} \mathrm{CO} 2$ eq). Em relação à categoria N-CA, o WC1 o estágio foi o responsável pelos maiores impactos, com 6,01 do total do sistema $-18,79 \mathrm{~kg} \mathrm{C}_{2} \mathrm{H}_{3} \mathrm{Cl}$ eq., seguido pelos RAs, com $5,47 \mathrm{~kg} \mathrm{C}_{2} \mathrm{H}_{3} \mathrm{Cl}$ eq.

Figura 2: Impactos de ACV da fase de construção do sistema usando IMPACT 2002+. A) Caracterização. B) Normalização. Onde: $\mathrm{Ca}=$ Carcinogênico; $\mathrm{N}-\mathrm{Ca}=$ Não cancerígeno; $I R=$ efeitos respiratórios (causados por inorgânicos); IR = radiação ionizante; $O D$ = redução do nível de ozônio; $R P=$ efeitos respiratórios (causados por orgânicos); $A E$ = Ecotoxicidade aquática; TE = Ecotoxicidade terrestre; TA- $N$ = Acidificação e Nutrição Terrestre; $L O=$ ocupação do solo; $A A$ = acidificação aquática; $A E$ = eutrofização aquática; $G W$ = aquecimento global; NRE = energía não renovável; $M E$ = Extração mineral.

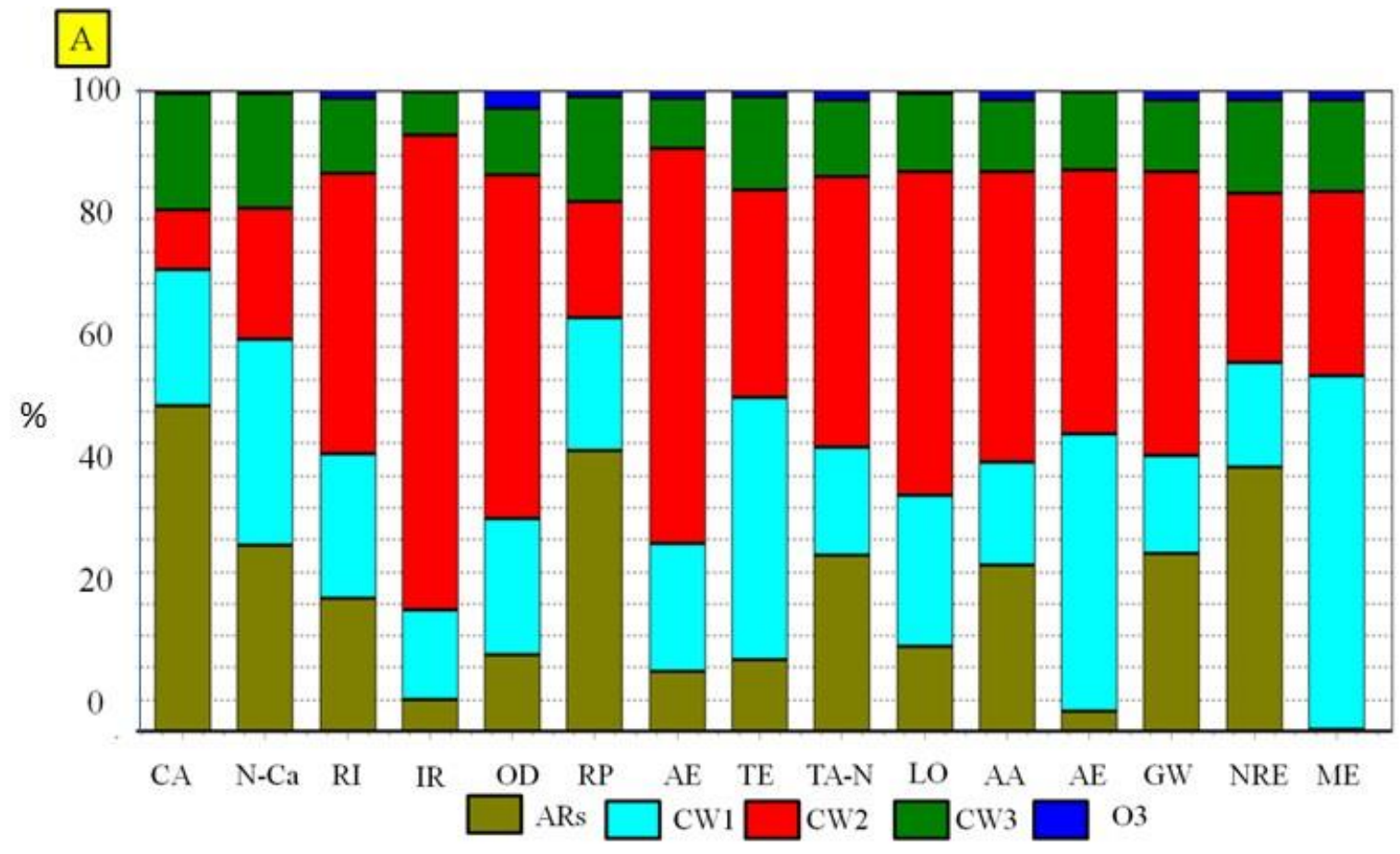




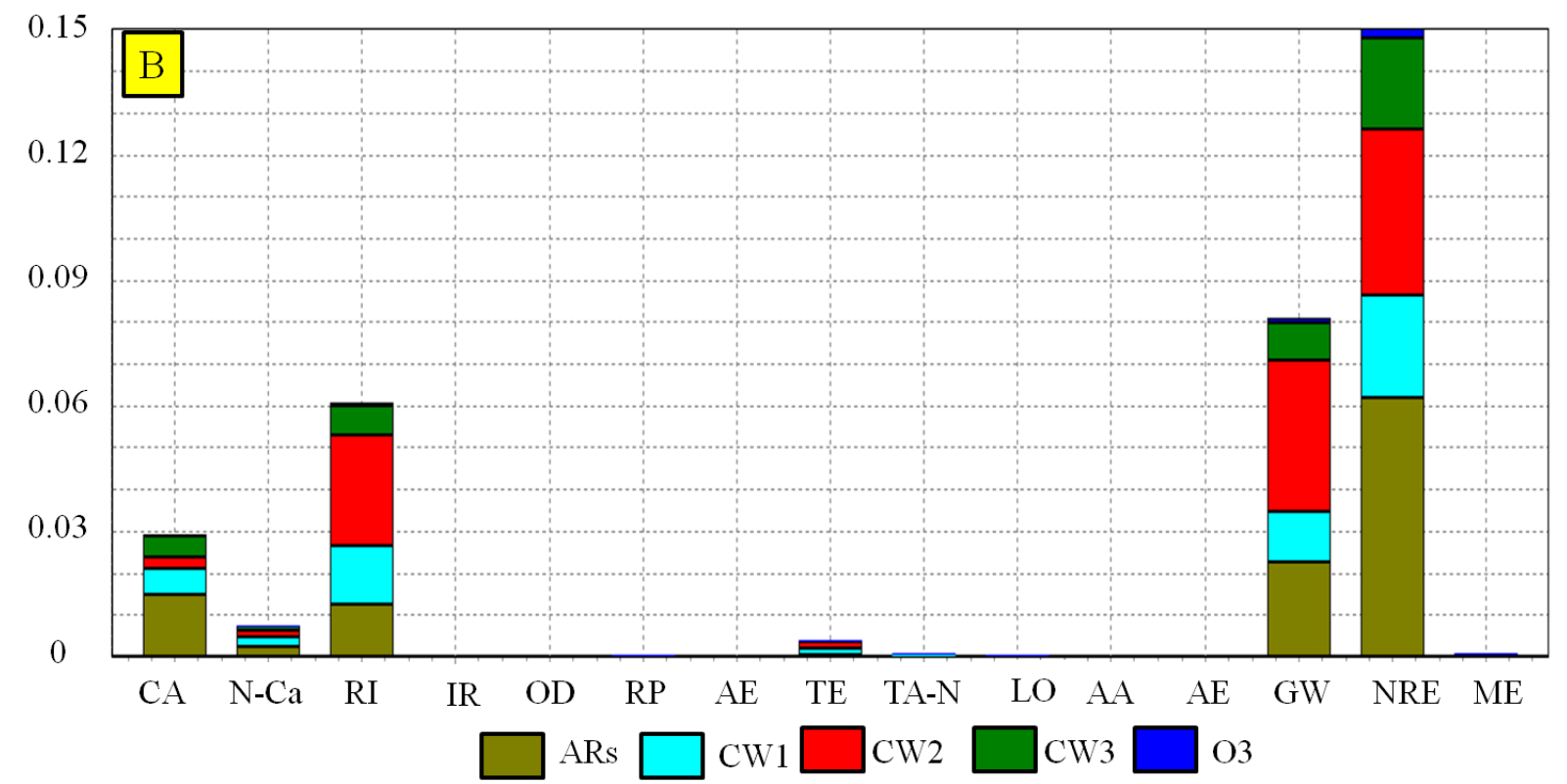

A Figura 3 apresenta a visão de rede da ACV realizada. Na visão de rede, o software apresenta os materiais que mais contribuíram para os impactos gerais de cada unidade de tratamento. Nesse contexto, quanto mais larga a linha vermelha entre as caixas, mais significativos são os impactos relacionados, enquanto os percentuais representam o quanto essa etapa contribui para os impactos totais dos sistemas.

Figura 3 - Vista da rede na ACV da fase de construção do Sistema RAs + WCs + $0_{3}$. Valor de 0,5\% de truncatura.

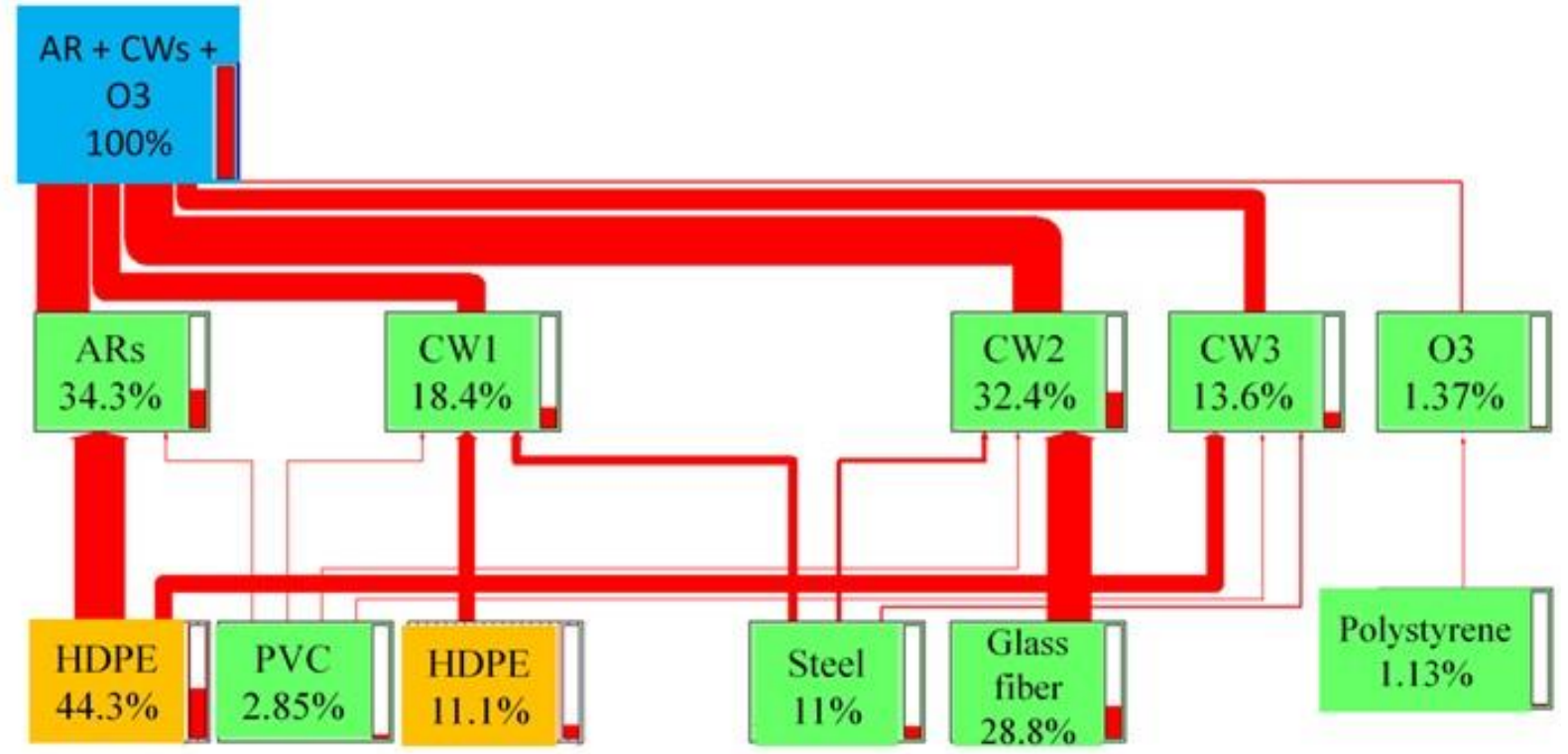

Conforme mostrado na Figura 3, os materiais que mais impactaram a produção do sistema foram o polietileno de alta densidade (PEAD), mais utilizado para os RAs e o plástico reforçado com fibra de vidro, aplicado no WC2. Resultados semelhantes foram encontrados por Resende et al. (2019) e Lutterbeck et al. (2017), que 
relataram que a maioria dos impactos da construção de sistemas WCs para tratamento de efluentes se deveu aos tanques de fibra de vidro. Porém, após comparar o cenário com tanques de fibra de vidro com o cenário com alvenaria (tijolos e aço), os autores concluíram que o potencial de causar impactos dos tanques de fibra de vidro era menor do que os materiais de alvenaria. No entanto, na hora de escolher os melhores materiais para a construção do sistema de tratamento, é importante verificar a disponibilidade de diferentes materiais na região onde o sistema será construído, com base também na logística reversa de cada um dos materiais de acordo com a economia local.

As unidades RAs e WC2 foram responsáveis por mais da metade dos impactos gerais da construção (34,3 e 32,4, respectivamente). Já a unidade de ozonização $\left(0_{3}\right)$ foi responsável por apenas $1,37 \%$ dos impactos da construção. Embora as caixas WC1, WC2 e WC3 tivessem o mesmo peso (40 kg cada), a aplicação das caixas de PEAD resultou em impactos menores do que o tanque de fibra de vidro. Nesse contexto, a substituição do tanque de fibra de vidro por caixas de PEAD pode ser uma alternativa para minimizar os impactos construtivos. Além disso, Lutterbeck et al., (2017) afirma que outra possibilidade de reduzir a pressão ambiental das unidades anaeróbias é recuperar energia da biometanização, bem como enxofre das emissões de $\mathrm{H}_{2} \mathrm{~S}$. Nesse caso, existe a possibilidade de ser recuperado como enxofre elementar sob um sistema de redução cíclica quando $\mathrm{FeCl}_{2}$ é aplicado para capturá-lo como pré-tratamento para um queimador de gás (SZARBLEWSKI et al., 2012).

Corominas et al., (2013) com tratamentos de baixa tecnologia, incluindo CWs, mostraram que a fase de construção pode ser responsável por até $80 \%$ dos impactos ambientais negativos. No entanto, apesar de alguns efeitos negativos, o desempenho ambiental dos CWs é muito melhor em comparação com outras tecnologias de tratamento, principalmente por se tratarem de sistemas descentralizados de tratamento de efluentes, que também permitem o tratamento de lodo in loco, evitando o transporte de lodo.

\subsection{ACV da operação do sistema RAs + WCs + $\mathrm{O}_{3}$}

A presente seção traz os resultados do desempenho ambiental quanto à operação do sistema integrado. Os benefícios ambientais, bem como os impactos diretos são apresentados nas Figuras 4 (A e B), principalmente no que diz respeito à remoção de poluentes que podem potencialmente reduzir os níveis de oxigênio na água e aumentar as condições de eutrofização. Nesse contexto, a ACV é apontada como uma importante ferramenta para avaliar os ganhos e efeitos negativos ambientais de cada etapa do sistema combinado RAs $+\mathrm{WCs}+\mathrm{O}_{3}$. 
Figura 4. Impactos do RAs + WCs + $\mathrm{O}_{3}$. A) Caracterização dos impactos. B) Impacta a normalização e a visualização da rede. Onde: $\mathbf{C a}=$ Carcinogênico; $\mathbf{N}-\mathrm{Ca}=$ Não cancerígeno; $I R=$ efeitos respiratórios (causados por inorgânicos); IR = radiação ionizante; $O D=$ redução do nível de ozônio; $R P=$ efeitos respiratórios (causados por orgânicos); $A E$ = Ecotoxicidade aquática; TE = Ecotoxicidade terrestre; TA-N = Acidificação e Nutrição Terrestre; $L O=$ ocupação do solo; $A A=$ acidificação aquática; $A E$ = eutrofização aquática; $G W=$ aquecimento global; NRE = energía não renovável; ME: Extração mineral.
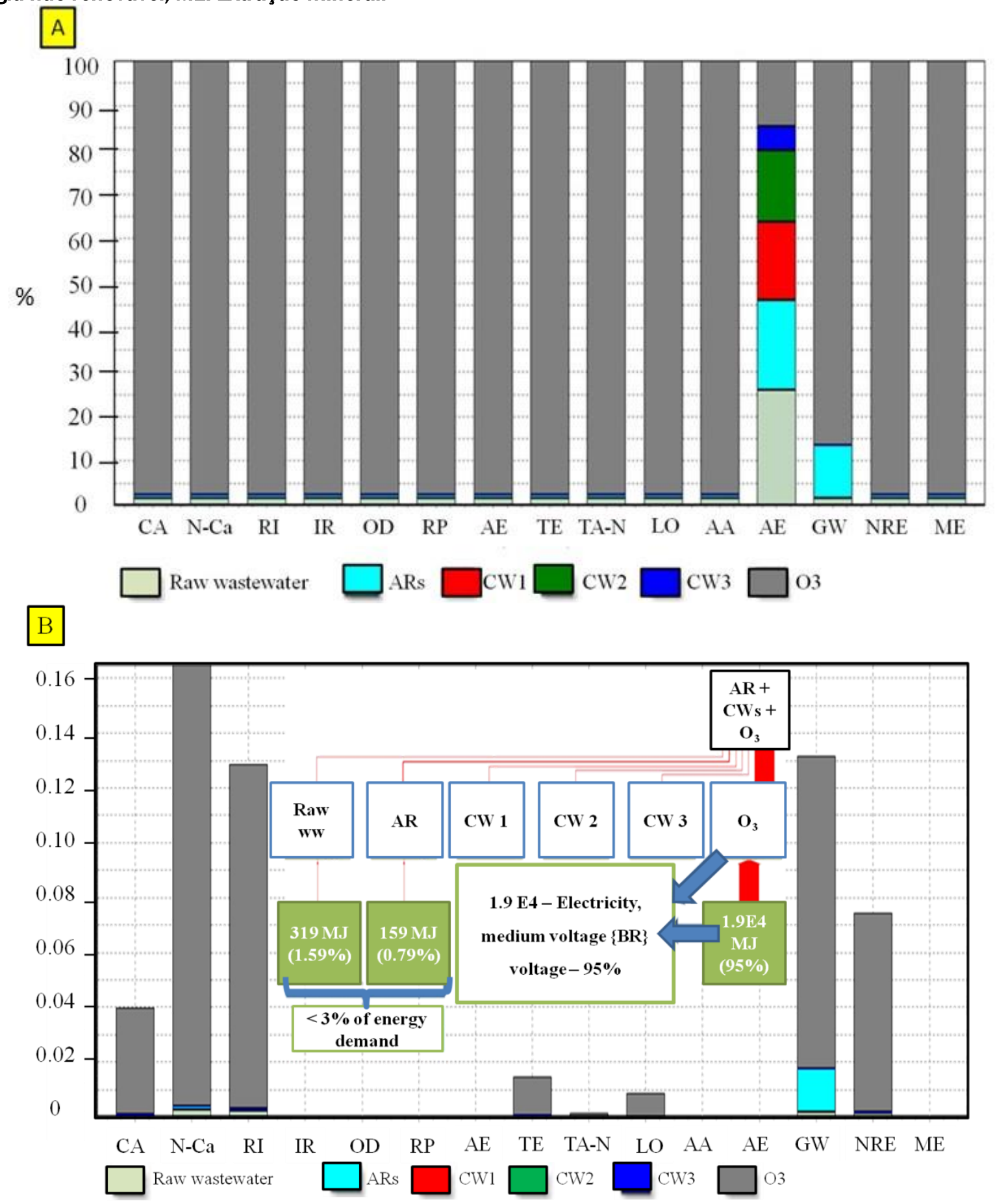

Conforme mostrado no gráfico de caracterização de impactos (Figura 4A), para quase todas as categorias de impacto (com exceção da eutrofização aquática) a unidade de ozonização foi o estágio mais significativo do 
sistema. Em relação à eutrofização aquática (EA), o despejo de esgoto bruto (sem nenhum tratamento) seria o responsável pelo mais significativo, devido às suas altas concentrações de fósforo e nitrogênio. Em relação ao impacto do GW, a unidade de RAs também apresentou alguma importância. Isso se deve principalmente à produção de biogás e, portanto, às emissões de metano do processo de digestão anaeróbia presente nesta etapa. Resultados semelhantes foram obtidos em estudos anteriores (LUTTERBECK et al., 2017, LOPES et al., 2020). A última etapa (unidade de ozonização) apresentou impactos menores que as outras unidades quanto à eutrofização devido à eficiência do tratamento na remoção de nutrientes, tanto $N$ quanto $P$.

Os dados de caracterização indicaram que o consumo de energia de média tensão foi o aspecto impactante do sistema, enquanto a eutrofização aquática (EA) apresentou-se como o impacto mais expressivo associado às cargas poluentes presentes nas águas residuárias. É importante destacar que $95 \%$ dos impactos foram relacionados ao processo de ozonização. Assim, mesmo considerando que cerca de $67 \%$ da matriz energética brasileira provém de usinas hidrelétricas (EPE, 2019), o uso de outras fontes de energia não ecologicamente corretas, como termelétricas, pode elevar consideravelmente os impactos ambientais negativos do sistema de tratamento.

Ao analisar os resultados da Normalização (Fig. 4B), pode-se observar que sete diferentes categorias foram as categorias mais impactadas: CA, N-Ca, RI, TE, LO, GW e NRE. Como já mencionado, o gerador $\mathrm{O}_{3}$ foi a unidade de maior impacto do sistema integrado devido às altas demandas de energia. Lutterbeck et al. (2020) afirma que o uso de tratamentos com alta necessidade de energia elétrica pode elevar significativamente os encargos ambientais do sistema. De acordo com Li et al. (2019), em alguns casos, os benefícios relativos das reduções de toxicidade são menores do que os encargos trazidos pela introdução de processos avançados de tratamento de águas residuais por causa da toxicidade indireta gerada durante a produção adicional de eletricidade, bem como contribuições da eliminação de materiais. Portanto, pode-se considerar a utilização de um pós-tratamento (unidade de polimento) utilizando processos de oxidação avançada, como a ozonização. No entanto, essa decisão deve ocorrer somente após uma análise cuidadosa dos eventuais benefícios e finalidades para as águas residuais tratadas, por exemplo, para descargas de vasos sanitários, lavagem de calçadas, irrigação de lavouras, entre outros.

\subsection{Avaliação econômica}

A avaliação econômica foi estimada usando 20 anos de vida para o sistema combinado considerando cada uma das etapas (Tabela 3) e de empresas locais que fornecem os materiais e equipamentos necessários para a construção e operação do sistema. Em relação aos custos, a unidade de ozonização apresentou os maiores gastos tanto na fase de construção quanto na operação, principalmente em função da aquisição do gerador de ozônio e da elevada demanda de energia elétrica. 
Tabela 3. Custos de construção e operação fornecidos por empresas locais para cada unidade do sistema combinado, considerando 20 anos de vida útil, tratando 100 L / semana de esgoto.

\begin{tabular}{|c|c|c|c|c|c|c|c|}
\hline & Unidade & RA & WC1 & WC2 & WC3 & $\mathrm{O}_{3}$ & Total \\
\hline \multicolumn{8}{|l|}{ Construção } \\
\hline $\begin{array}{c}\text { Materiais } \\
\text { Mão de Obra de }\end{array}$ & US\$ & 195 & 46 & 58 & 69 & 313 & 681 \\
\hline construção & US\$ & 80 & 40 & 40 & 60 & - & 220 \\
\hline $\begin{array}{l}\text { Operação } \\
\text { Demanda de }\end{array}$ & & a? & $A G$ & & & 1077 & 1000 \\
\hline Água & US\$ & $\begin{array}{c}9,2 \\
-\end{array}$ & $\begin{array}{c}4.6 \\
-\end{array}$ & - & - & $\begin{array}{l}10 / 5 \\
182\end{array}$ & $\begin{array}{c}1089 \\
182\end{array}$ \\
\hline $\begin{array}{l}\text { Colheita da } \\
\text { Biomassa }\end{array}$ & US\$ & - & 200 & - & 200 & - & 400 \\
\hline Remoção de lodo & US\$ & 400 & - & - & - & - & 400 \\
\hline Total & US\$ & 684,2 & 290,8 & 97,5 & 328,9 & 1752 & 2972 \\
\hline
\end{tabular}

Considerando apenas os RAs para o tratamento de efluentes, os custos de construção e operacionais para o tratamento de efluentes urbanos foram estimados em 6,5 US \$ / $\mathrm{m}^{3}$. Ao integrar ARs + CWs híbridos, os custos basicamente dobraram, aumentando para cerca de 13,5 US $\$ / \mathrm{m}^{3}$. Porém, se a unidade de ozonização também for considerada no tratamento, os custos globais do sistema combinado foram estimados em 26,8 US $\$ / \mathrm{m}^{3}$, afetando diretamente a viabilidade do sistema combinado nas condições estudadas. Nesse contexto, alternativas de geração de energia devem ser fornecidas de forma a promover a aplicação de ozonização e outros processos de oxidação avançados.

\section{CONCLUSÃO}

Este estudo é uma nova tentativa de quantificar a sustentabilidade ambiental e os custos econômicos separadamente da construção e operação de um sistema combinado composto de RA + WCs $+\mathrm{O}_{3}$ para 0 tratamento e reuso local de águas residuais urbanas geradas em uma casa ou em uma pequena comunidade , a fim de identificar as etapas de tratamento mais significativas em termos de impactos ambientais e de viabilidade econômica, e propor aspectos de investigação mais aprofundada para a melhoria geral do sistema combinado.

No que se refere à construção do sistema, a maioria dos impactos está relacionada à construção dos estágios RA e CW2, devido à aplicação de polietileno de alta densidade e fibra de vidro. 0 primeiro foi o maior responsável pelas emissões de $\mathrm{CA}\left(37,4 \mathrm{~kg}\right.$ dos $73,4 \mathrm{~kg}$ de $\mathrm{CH}_{2} \mathrm{CH}_{3} \mathrm{Cl}$ eq) e $\mathrm{NRE}\left(9,42 \mathrm{E}^{3}\right.$ de $2,28 \mathrm{E}^{4} \mathrm{MJ}$ primário), enquanto o CW2 apresentou os maiores impactos em relação ao RI (0,268 de 0,614 kg PM2,5 eq ) e GW (356 de $803 \mathrm{~kg} \mathrm{CO} 2 \mathrm{eq})$.

Na operação de tratamento, a etapa de ozonização foi responsável por 95\% dos impactos ambientais, devido ao alto consumo de energia. Esses impactos podem ser drasticamente reduzidos por meio da geração local de energia renovável. 
Em relação à avaliação econômica, o consumo de energia foi responsável pela maior parte dos custos (US\$ 1089,00), seguido pelo manejo do lodo (US \$ 400) e coleta de biomassa (US\$ 400). Na construção do sistema, o gerador de ozônio respondeu por quase um terço dos custos totais (313 de US\$ 901).

Embora o custo do tratamento encontrado no presente $\left(28,6\right.$ US $\left.\$ / \mathrm{m}^{3}\right)$ tenha sido bem superior a outros estudos da literatura, diferentemente deles também foi abordado o reuso da água e a aplicação de uma etapa de tratamento $\left(\mathrm{O}_{3}\right)$ para garantir a desinfecção total e desintoxicação das águas residuais.

\section{AGRADECIMENTOS}

Gustavo S. Colares agradece a comissão de aperfeiçoamento de pessoal de nível superior da CAPES código de apoio ao financiamento - 001. Ênio Leandro Machado agradece o apoio financeiro da FAPERGS “Fundação de Amparo à Pesquisa do Estado do Rio Grande do Sul, PqG2017, projeto cnpq-9 307257/ 2015-0 e projeto CNPq 307599 / 2018-3. Patrícia Cerentini e Gabriela Paranhos agradecem aos apoios financeiros das bolsas CNPq e FAPERGS com administração da UNISC.

\section{REFERÊNCIAS}

ÁlVAREZ, J. A., ÁVILA, C., OTTER, P., KILIAN, R., ISTENIČ, D., ROLLETSCHEK, M. \& JORGENSEN, C. Constructed wetlands and solar-driven disinfection technologies for sustainable wastewater treatment and reclamation in rural India: SWINGS project. Water Sci. Technol., 76(6), 1474-1489. 2017. DOI: 10.2166/wst.2017.329.

ALZEYADI, A., AL-ANSARI, N., LAUE, J., \& ALATTABI, A. Study of biomass bottom ash efficiency as phosphate sorbent material. Civil Engineering Journal, 11(5), 2392-2401. 2019. DOI: 10.28991/cej-2019-03091419.

ABNT NBR 13969: Septic tank - Units for treatment and disposal of liquid effluents - Project, construction, and operation. Rio de Janeiro, 1997.

COLARES, G. S., DE SOUZA CELENTE, G., DA SILVA, F. P., DE LORETO, A. C., LUTTERBECK, C. A., KIST, L. T., \& MACHADO, $\hat{E}$. L. Combined system for the treatment and reuse of urban wastewater: the efficiency of anaerobic reactors+ hybrid constructed wetlands+ ozonation. Water Sci. Technol. 80(2), 254-264. 2019. DOI: 10.2166/wst.2019.270.

COLARES, G. S., DELL'OSBEL, N., WIESEL, P. G., OLIVEIRA, G. A., LEMOS, P. H. Z., DA SILVA, F. P. LUTTERBECK, C.A., KIST, L.T. \& MACHADO, Ê. L. Floating treatment wetlands: A review and bibliometric analysis. Sci. Total. Environ. 136776. 2020. https://doi.org/10.1016/j.scitotenv.2020.136776.

COOPER, R. J., HAWKINS, E., LOCKE, J., THOMAS, T., \& TOSNEY, J. Assessing the environmental and economic efficacy of two integrated constructed wetlands at mitigating eutrophication risk from sewage effluent. Water and Environment Journal. 2020. DOI:10.1111/wej.12605.

COROMINAS, L., FOLEY, J., GUEST, J. S., HOSPIDO, A., LARSEN, H. F., MORERA, S., \& SHAW, A. Life cycle assessment applied to wastewater treatment: state of the art. Water Res. 47(15), 5480-5492. 2013. http://dx.doi.org/10.1016/j.watres.2013.06.049.

DE QUEIROZ, R. D. C. S., MARANDUBA, H. L., HAFNER, M. B., RODRIGUES, L. B., \& DE ALMEIDA NETO, J. A. Life cycle thinking applied to phytoremediation of dairy wastewater using aquatic macrophytes for treatment and biomass production. Journal of Cleaner Production, 122006. 2020. DOI: 10.1016/j.jclepro.2020.122006. 
DELL'OSBEL, N., MACHADO, Ê. L"Life Cycle Assessment of Urban Effluent Treatment Systems using Microalgae and Constructed Wetlands ". Avaliação do Ciclo de Vida de Sistemas de Tratamento de Efluentes Urbanos utilizando Microalgas e Wetlands Construídos. REMOA, 18(1), 1. 2019. DOI: 10.5902/2236130835929.

DELL'OSBEL, N., COLARES, G. S., DE OLIVEIRA, G. A., DE SOUZA, M. P., BARBOSA, C. V., \& MACHADO, Ê. L. Bibliometric Analysis of Phosphorous Removal Through Constructed Wetlands. Water Air Soil Poll. 231(3), 1-18. 2020. doi.org/10.1007/s11270-020-04513-1.

DE SOUZA CElENTE, G., COlARES, G. S., DA SILVA ARAÚJO, P., MACHADO, Ê. L., \& LOBO, E. A. Acute ecotoxicity and genotoxicity assessment of two wastewater treatment units. Environ. Sci. Pollut. R. 1-8. 2020. https://doi.org/10.1007/s11356-019-07308-y. 